\title{
High Capacity, Low Latency Data Transmission Using Hollow Core-Photonic Bandgap Fibers
}

\author{
N.V. Wheeler, Y. Chen, J.R. Hayes, S.R. Sandoghchi, G.T. Jasion, T.D. Bradley, E.N. Fokoua, N.K. Baddela, \\ D.R. Gray, Z. Liu, Y. Jung, S. U. Alam, R. Slavik, F. Poletti, M.N. Petrovich and D.J. Richardson. \\ Optoelectronics Research Centre, University of Southampton, Southampton, SO17 1BJ, U.K. \\ n.v.wheeler@soton.ac.uk
}

\begin{abstract}
We discuss our recent progress in hollow core-photonic bandgap fiber fabrication for high capacity transmission, focusing on two key areas: longitudinal uniformity and length upscaling as well as reviewing highlight results in data transmission.

OCIS codes: (060.5295) Photonic crystal fibers, (060.2330) Fiber optic communications
\end{abstract}

\section{Introduction}

Hollow core-photonic bandgap fibers (HC-PBGFs) deliver low loss optical guidance in an air core surrounded by a periodic array of air holes defined by a silica web. More than $99 \%$ of the light transmitted by a HC-PBGF travels in air, providing these fibers with a unique set of transmission properties; in particular, this fiber geometry offers ultralow non-linearity [1] and ultimate low latency [2], which, combined with the potential for lower attenuation than the current state-of-the-art conventional all-silica fibers, has led to a growing interest in the use of HC-PBGFs for data transmission. In particular, the $1.54 \mu \mathrm{s} / \mathrm{km}$ latency reduction offered by HC-PBGFs is attractive for applications which are time-sensitive, such as intra- and inter- connections in data centers.

This ambitious target application for HC-PBGFs has provided a significant driving force to improve several aspects of these fibers and led to substantial progress in recent years. This progress includes: development of fibers with record combinations of wide operating bandwidth and low loss through improved control of the core surround during fabrication [3], the first single mode high capacity transmission experiments at $1550 \mathrm{~nm}$ [4] and $2 \mu \mathrm{m}$ [5] (where the minimum loss of HC-PBGFs is believed to lie), the first multimode transmission experiment in a HCPBGF which led to a new capacity record of $73.7 \mathrm{~Tb} / \mathrm{s}$ [6], development of characterization techniques to provide extensive data on modal quality [7] and fabrication of narrow bandwidth, specialty single mode and polarization maintaining HC-PBGFs [8]. In this paper we review our latest results, focusing in particular on longitudinal uniformity and length upscaling as well as new record results in data transmission over long length HC-PBGFs.

\section{Length Upscaling: Increasing Fiber Yield}

It is evident from the literature that, until recently, the longest reported HC-PBGF was $\sim 1 \mathrm{~km}[9,10]$ and that most routinely these fibers are produced in shorter lengths, typically of a few hundred meters. For applications in telecommunications, the ability to make longer fiber lengths is essential both in terms of reach, to connect distant terminals, and also to increase the economic viability of this fiber solution. We identified three key challenges [11] to overcome in order to produce low loss, uniform, long lengths of HC-PBGFs: fiber breaks during the fiber draw; defects along the length of the fiber which locally increase the loss; and the introduction of distortions into the fiber structure due to a need to increase preform size to achieve longer lengths which requires an increased draw-down ratio [12].

Since the first report of fabrication of HC-PBGFs [13], the main elements of the fabrication process have remained unchanged; the fabrication follows a two-step 'stack and draw' process. In this process, up to several hundred, meter long capillaries ( millimeter diameter) are stacked into the required geometry to form the initial preform. The manual nature of this process, along with the huge surface area provided by the assembled capillaries, means that extreme care has to be taken to avoid contamination of the preform as this can then lead to defects (structural irregularities) in the final fiber [14]. These defects can lead to both fiber breaks and an increase in the overall loss in the fiber. Through improved cleanliness and glass handling and processing these defects can be eliminated from the fiber preform, removing two obstacles which prohibit fabrication of long fiber lengths.

In the two step stack and draw process, this initial preform is drawn first to canes (few millimeter diameter) and then subsequently to fiber. The third identified challenge originates from the need to produce larger diameter canes which then have a larger draw-down ratio to the final fiber. This leads to changes in the fluid dynamics of the glass in the 'neck-down' region of the preform which result in a need for a very precise control of the applied pressures to the core and cladding regions of the preform during the fiber draw in order to accurately counter surface tension 
effects [12]. To fully understand this mechanism we employed fluid dynamics modelling and subsequently developed the required level of pressure control to overcome this final challenge.

Through this improved process control and understanding we fabricated new record lengths of HC-PBGF. These new fibers were fabricated with a 19-cell defect core, introduced during the stacking process by removing 19 elements from the center of the preform and replacing them with a 'core tube' (a large capillary) to support this core region. The use of the core tube increased the fiber yield and also through careful design, enabled fabrication of a final fiber with a record wide operating bandwidth. Two key highlights of this work are a $3.85 \mathrm{~km}$ length operating at $2 \mu \mathrm{m} \mathrm{[15]} \mathrm{and} \mathrm{an} 11 \mathrm{~km}$ length operating at $1.55 \mu \mathrm{m} \mathrm{[16];} \mathrm{representing} \mathrm{an} \mathrm{order} \mathrm{of} \mathrm{magnitude} \mathrm{improvement} \mathrm{on}$ the previous state-of-the-art.
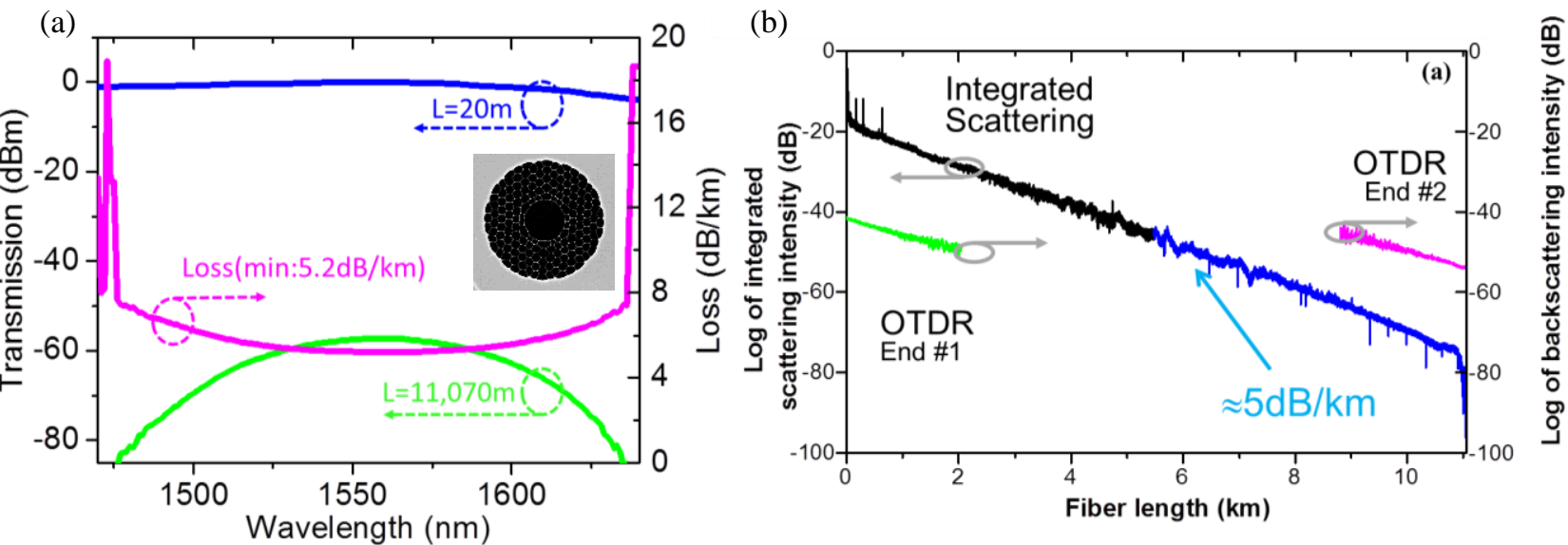

Figure 1: (a) SEM image of record length HC-PBGF, (b) Cutback loss measurement from $11.07 \mathrm{~km}$ to $20 \mathrm{~m}$, (c) Integrated out-scattered intensity as a function of fiber length. The black and blue traces are measurements obtained from opposite ends of the fiber. OTDR measurements are also shown (pink and green traces, plotted with an arbitrary offset for clarity), highlighting the shorter measurement reach and less accurate loss estimate $(\sim 4 \mathrm{~dB} / \mathrm{km})$.

A scanning electron microscope (SEM) image of the cross-section of the $11 \mathrm{~km}$ fiber is shown inset to Fig. 1a; the core diameter is $\sim 30 \mu \mathrm{m}$, the pitch (hole-to-hole spacing, $\Lambda$ ) is $6.2 \mu \mathrm{m}$, the relative hole size $(\mathrm{d} / \Lambda)$ is 0.992 and the average cladding strut thickness is $50 \mathrm{~nm}$. The fiber attenuation (Fig. 1a) was initially found through a cutback measurement from $11.07 \mathrm{~km}$ to $20 \mathrm{~m}$ and shows a minimum loss of $5.2 \mathrm{~dB} / \mathrm{km}$, indicating high uniformity of the fiber along the complete length. From a shorter length cutback (not shown) the surface mode free $3 \mathrm{~dB}$ operating bandwidth of the fiber was found to exceed $200 \mathrm{~nm}$ which represents a new record for a 19-cell HC-PBGF operating at $1550 \mathrm{~nm}$.

As we have worked to increase the length of HC-PBGFs and to reduce the fiber attenuation it has been necessary to exploit characterization tools typically used to analyze conventional fibers as well as to develop new tailored techniques. In particular, longitudinal uniformity has become increasingly important, which, in conventional fibers, is typically analyzed using OTDR. The $11 \mathrm{~km}$ fiber was initially probed using OTDR but due to the limited dynamic range of the instrument, this technique can only deliver information on the first $\sim 2 \mathrm{~km}$ of the fiber, making it apparent that a specialized tool is required. To this end, we designed and built a new technique referred to as Optical Side Scattering Radiometry (OSSR) [17]. In this technique, light from an amplified diode laser (at $1560 \mathrm{~nm}$ ) is coupled into the fiber while an integrating sphere measures the light scattered out of the fiber as it is passed through it. The results of OSSR analysis on the $11 \mathrm{~km}$ fiber are shown in Fig. 1c where, to cover the entire fiber length with the best signal-to noise ratio, the fiber was analyzed bi-directionally. In this trace, it would be expected that for a perfectly uniform fiber length, the out-scattered power would decrease linearly with fiber length. In Fig. 1c it is clear that for the $11 \mathrm{~km}$ length this is generally the case, indicating a highly uniform fiber. The small ripples in the data, indicating small changes in out-scattered power, were found to account for $<1 \mathrm{~dB}$ extra loss contribution over the full length and the slope of the trace indicates a fiber attenuation of $5.14 \mathrm{~dB} / \mathrm{km}$, in very good agreement with the cutback measurement. This OSSR has proved to be a very powerful tool for longitudinal fiber analysis, providing a dynamic range of $\sim 60 \mathrm{~dB}$ in combination with high resolution $(\sim 5 \mathrm{~cm})$.

\section{Data transmission}

Following demonstration of the low attenuation and high longitudinal uniformity of the record length $11 \mathrm{~km}$ fiber, data transmission at $10 \mathrm{~Gb} / \mathrm{s}$ (direct detection) and 20Gbit/s (coherent detection) was demonstrated [11]. Due to the 
wide operating bandwidth of the fiber, a limiting factor in the achievable transmission capacity was the bandwidth of the available amplifiers (EDFAs). Direct detection permitted the lowest overall latency ( $>15 \mu \mathrm{s}$ reduction compared to standard fiber), a key feature of this fiber format. The only penalties observed were due to the fiber loss; through increase of our understanding through extensive numerical and fluid dynamics modelling we believe we can tackle this challenge.

\section{Conclusions and Outlook}

We have reviewed recent progress in fabrication of record HC-PBGF lengths, up to $11 \mathrm{~km}$, with highly uniform attenuation, confirmed through development of a new technique for longitudinal analysis of optical fibers. These lengths are $\sim$ an order of magnitude increase over previous reports and represent a step-change in the continuing work towards the use of HC-PBGFs for data transmission. This is highlighted in Fig. 2a where we use a new metric (combining fiber length, attenuation and bandwidth) to highlight progress in recent years. Ongoing efforts are now directed towards combining this remarkable increase in fiber yield with further loss reduction to achieve ultralow loss, long length, longitudinally uniform HC-PBGFs. From extensive numerical modelling we have found that a 37cell defect HC-PBGF with a $40 \mu \mathrm{m}$ core operating in the $2 \mu \mathrm{m}$ region has a minimum loss of $0.2 \mathrm{~dB} / \mathrm{km}$; this can be achieved without any reduction in surface roughness. This structure and its expected transmission loss is shown is Fig. 2b. To achieve this ultralow loss, structural uniformity, in particular in the core surround region is essential and as such precise control of this during the fabrication process will be crucial.
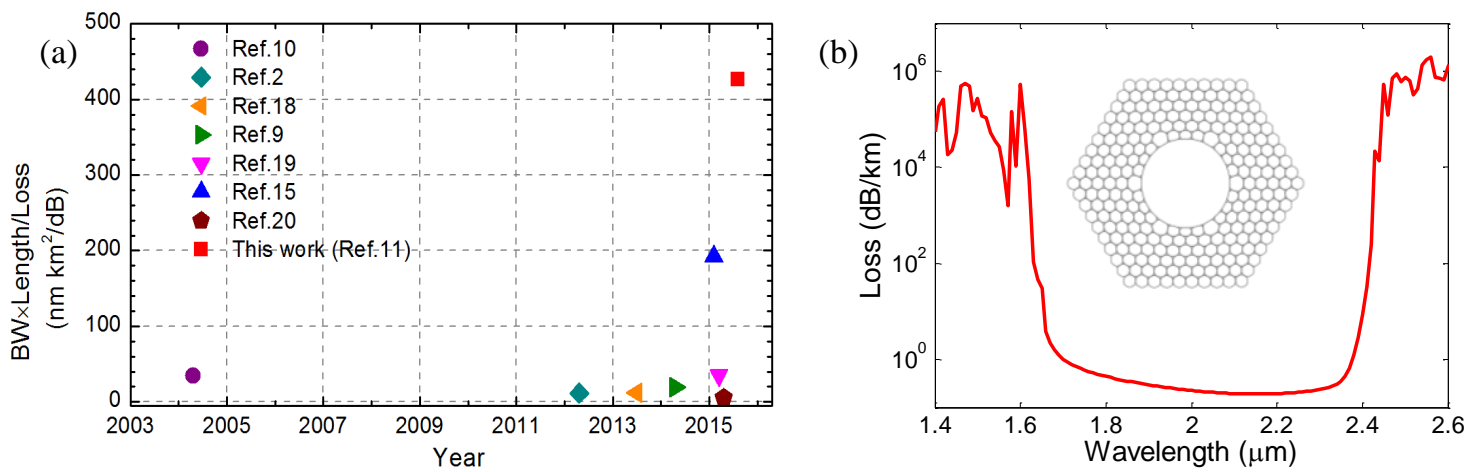

Figure 2: (a) Best performing HCPBGFs reported to date are compared using a length $\times B W /$ loss figure of merit

[11] (b) Predicted loss for optimized 37-cell HC-PBGF (inset) with a minimum loss of $0.2 \mathrm{~dB} / \mathrm{km}$ at $\sim 2 \mu \mathrm{m}$.

The authors gratefully acknowledge Brian J. Mangan (OFS Labs, Somerset, NJ, 08873, USA) for provision of a core tube element which was used in fibre fabrication. This work was funded by EU FP7 through project MODE-GAP (grant agreement 258033) and the UK Engineering and Physical Sciences Research Council (EPSRC) through grants EP/H02607X/1 (Centre for Innovative Manufacturing in Photonics) and EP/I061196X (HYPERHIGHWAY).

\section{References}

[1] X. Peng et al., Opt. Express, 19(2) pp. 923-932, 2011

[2] N. V. Wheeler et al., National Fiber Optic Engineers Conference, OSA Technical Digest (Optical Society of America, 2012), paper PDP5A.2

[3] F. Poletti et al., Nat. Photonics, 7(4) pp. 279-284, 2013.

[4] R. Slavík et al., European Conference on Optical Communication (ECOC) Amsterdam 16-20 Sep 2012 Mo.2.F.2

[5] M. N.Petrovich et al. Opt. Express 21(23) pp.28559-28569 2013

[6] Y. Jung et al., Optical Fiber Communication Conference, OSA Technical Digest (online) (Optical Society of America, 2013), PDP5A.3.

[7] D. R.Gray et al. Opt. Express 23(8) pp.10540-10552 2015

[8] B. J. Mangan et al., Proceedings of European Conference on Optical Communication (ECOC 2015), paper \#ID-0721

[9] R. van Uden et al., Optical Fiber Communication Conference, OSA Technical Digest (online) (Optical Society of America, 2014), W4J.3.

[10] B.J. Mangan, et al., Optical Fiber Communication Conference Proc. OFC 2004, PD24

[11] Y. Chen et al. Journal of Lightwave Technology pp.1-10 2015

[12] G. T. Jasion et al., Optical Fiber Communication Conference, OSA Technical Digest (online) (Optical Society of America, 2015), W2A.37.

[13] R. F. Cregan et al., "Single-Mode Photonic Band Gap Guidance of Light in Air," Science 285(5433) pp. 1537-1539 1999.

[14] S. R. Sandoghchi et al., Optical Fiber Communication Conference, OSA Technical Digest (online) (Optical Society of America, 2014),

M2F.6.

[15] Z. Liu et al., J. Lightwave Technol., 33(7) pp. 1373-1379, 2015.

[16] Y. Chen et al., Optical Fiber Communication Conference Post Deadline Papers, OSA Technical Digest (online) (Optical Society of

America, 2015), paper Th5A.1.

[17] S. R. Sandoghchi et al., Proceedings of European Conference on Optical Communication (ECOC 2015), paper \#ID-568.

[18] M. H. Frosz et al. Opt. Lett., 38(3) pp. 2215-2217, 2013.

[19] H. Zhang et al. Opt. Express, 23(4) pp. 4946-4951, 2015.

[20] B. J. Mangan et al., Optical Fiber Communication Conference, OSA Technical Digest (online) (Optical Society of America, 2015), M3D.4. 